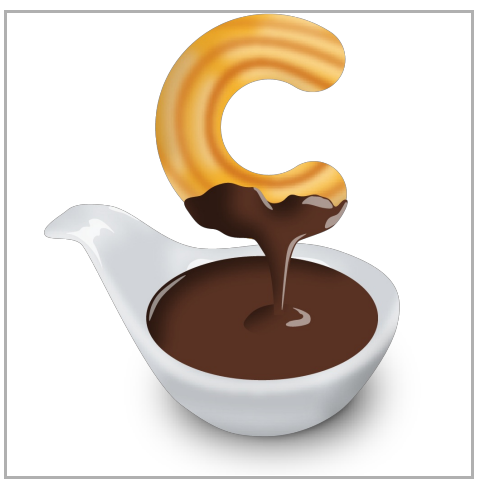

(3) Isolation and Fixation of Nuclei from the Mouse Brain for Dip-C

\title{
Longzhi $\operatorname{Tan}^{1}$
}

${ }^{1}$ Stanford University

NOV 18, 2020

\section{Longzhi Tan}

Stanford University

\section{open ठaccess}

\section{DOI:}

dx.doi.org/10.17504/protocol s.io.bpsxmnfn

\section{Protocol Citation: Longzhi}

Tan 2020. Isolation and

Fixation of Nuclei from the Mouse Brain for Dip-C.

\section{protocols.io}

https://dx.doi.org/10.17504/p rotocols.io.bpsxmnfn

License: This is an open access protocol distributed under the terms of the Creative Commons Attribution License, which permits unrestricted use, distribution, and reproduction in any medium, provided the original author and source are credited

Protocol status: Working We use this protocol and it's working

Created: Nov 17, 2020

Last Modified: Nov 18, 2020

PROTOCOL integer ID: 44599

\section{Reagents}

\section{Prepare $1.5 \mathrm{M}$ sucrose (I $40 \mathrm{~mL}$ ):}


- I $20.538 \mathrm{~g} \& 8$ sucrose Merck MilliporeSigma (Sigma-Aldrich) Catalog \#84097 (final: [M] 1.5 Molarity (M) , [M] 51.3 Mass / \% volume )

- I $40 \mathrm{~mL}$ water

- Heat and vortex to mix.

- Filter to sterilize.

- Store at $8^{\circ} 4^{\circ} \mathrm{C}$

2 Prepare Nuclei Isolation Medium 1 ( $\triangle 45 \mathrm{~mL}$; note that Tris is replaced with HEPES):

- \ $7.5 \mathrm{~mL} 1.5 \mathrm{M}$ sucrose (final: [M] 250 millimolar (mM), [M] 8.56 Mass / \% volume )

- I $562.5 \mu \mathrm{L} \& 8 \mathrm{KCl}(2 \mathrm{M}), \mathrm{RNase}$-free Thermo Fisher Catalog \#AM9640G (final: [M] 25 millimolar (mM) )

- I $450 \mu \mathrm{L} \& 8$ HEPES (1 M) Thermo Fisher Catalog \#15630080 (final: [M] 10 millimolar (mM)

- \ $225 \mu \mathrm{L} \quad \& 81 \mathrm{M} \mathrm{MgCl}$ Invitrogen - Thermo Fisher Catalog \#AM9530G (final: [M] 5 millimolar (mM)

- I $36.2625 \mathrm{~mL}$ water

- Vortex to mix.

- Store at $8^{\circ} 4^{\circ} \mathrm{C}$

3 Prepare $1 \%$ BSA in PBS ( I $1.2 \mathrm{~mL}$ per sample; recipe below for $\boldsymbol{\Delta} 10 \mathrm{~mL}$ ):

- $80.1 \mathrm{~g}$ 88 Bovine Serum Albumin (BSA) Fraction V-Molecular Biology Grade Gemini BioProducts Catalog \#700-106P

- I $10 \mathrm{~mL} \& 8$ PBS, pH 7.4 Thermo Fisher Catalog \#10010023

- Vortex to mix.

- Rotate until fully dissolved.

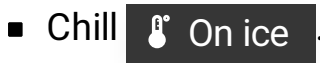

- Store at $8^{\circ}-20^{\circ} \mathrm{C}$ if needed.

\section{Nuclei Isolation}

4 Chill a

88 KIMBLE 2mL Glass Dounce Tissue Grinder Set Merck MilliporeSigma (SigmaAldrich) Catalog \#D8938

(or larger sizes:

89 KIMBLE Dounce tissue grinder set $7 \mathrm{~mL}$ complete Merck MilliporeSigma (SigmaAldrich) Catalog \#D9063 


\section{KIMBLE Dounce tissue grinder set $15 \mathrm{~mL}$ complete Merck MilliporeSigma (Sigma-}

Aldrich) Catalog \#D9938

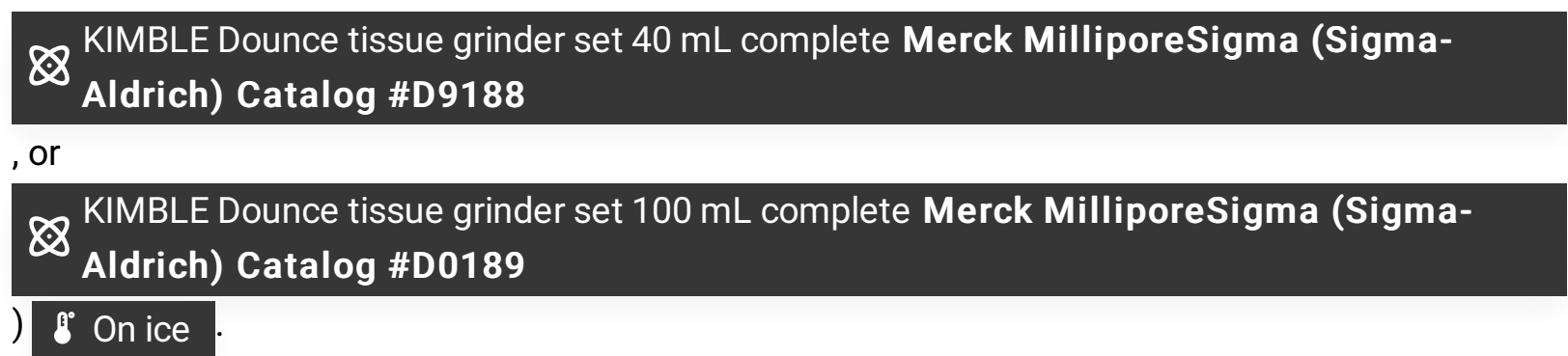

5 Prepare $1 \mathrm{mM} \mathrm{DTT}$ :

- $\triangle 1 \mathrm{~mL}$ water

- I $1 \mu \mathrm{L}$

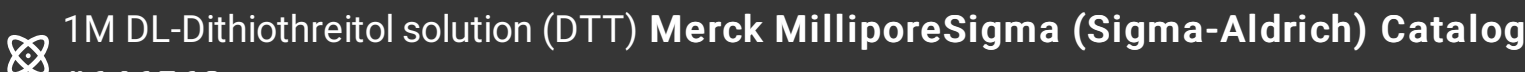
\#646563

(aliquoted and stored at $8^{\circ}-20^{\circ} \mathrm{C}$ )

- Vortex to mix.

6 Prepare Nuclei Isolation Buffer without Triton ( $\triangle 6 \mathrm{~mL}$ per sample):

- $\triangle 6 \mathrm{~mL}$ Nuclei Isolation Medium 1

- I $6 \mu \mathrm{L} 1 \mathrm{mM} \mathrm{DTT}$ (final: [M] 5 micromolar $(\mu \mathrm{M})$

- Vortex to mix.

- Chill $8^{\circ}$ On ice

7 Prepare Nuclei Isolation Buffer with Triton ( $12 \mathrm{~mL}$ per sample):

- $\quad 2 \mathrm{~mL}$ Nuclei Isolation Buffer without Triton

- $\triangle 20 \mu \mathrm{L}$

88 Triton X-100, 10\% solution Merck MilliporeSigma (Sigma-Aldrich) Catalog \#93443 (final: [M] $0.1 \%(\mathrm{~V} / \mathrm{v})$

- Vortex to mix.

- Chill $\mathfrak{f}^{\circ}$ On ice

8 Add $\triangle 2 \mathrm{~mL}$ ice-cold Nuclei Isolation Buffer with Triton to the homogenizer.

9 Dounce the tissue with 5 strokes of the loose pestle (A), and 15 strokes of the tight pestle (B). 
10 Transfer the homogenate to a tube.

11 Centrifuge at $\because 100 \times \mathrm{g}, 4^{\circ} \mathrm{C}, 00: 08: 00$

12 Carefully remove supernatant without disrupting the soft pellet.

13 Resuspend in $\triangle 2 \mathrm{~mL}$ Nuclei Isolation Buffer without Triton.

14 Centrifuge at $\because 100 \times \mathrm{g}, 4^{\circ} \mathrm{C}, 00: 08: 00$

15 Carefully remove supernatant without disrupting the soft pellet.

16 Resuspend in $\triangle 2 \mathrm{~mL}$ Nuclei Isolation Buffer without Triton.

17 Filter with $\& 8$ Falcon $40 \mu \mathrm{m}$ Cell Strainer Corning Catalog \#352340 or

88 Corning $^{\text {TM }}$ Falcon $^{\text {TM }}$ Test Tube with $35 \mu m$ Cell Strainer Snap Cap Corning Catalog \#352235 
18 To every $\triangle 1 \mathrm{~mL}$ of cells, add $\triangle 66.7 \mu \mathrm{L}$

88 Paraformaldehyde 32\% (methanol free) Electron Microscopy Sciences Catalog \#15714 (final: [M] 2 Mass Percent ).

19 Rotate at $8^{\circ}$ Room temperature for $\$ 0: 10: 00$

20 Add \ $200 \mu \mathrm{L}$ 1\% BSA in PBS.

21 Invert to mix.

22 Centrifuge at $\because 1000 \times \mathrm{g}, 4^{\circ} \mathrm{C}, 00: 05: 00$

23 Remove supernatant.

24 Resuspend in $\triangle 1 \mathrm{~mL}$ ice-cold $\underline{1 \% \text { BSA in PBS. }}$

25 Count with 88 C-Chip disposable hemacytometer INCYTO Catalog \#DHC-N01 , and aliquot to up to 0.5 million cells per tube. 
26 Centrifuge at $\because 1000 \times \mathrm{g}, 4^{\circ} \mathrm{C}, 00: 05: 00$

27 Remove supernatant.

28 Store at $8^{\circ}-80^{\circ} \mathrm{C}$ 